\title{
Revealing Soil Organic Matter-mineral Associations with Advanced Chemical Imaging Methods
}

Alice Dohnalkova ${ }^{1}$, Libor Kovarik ${ }^{1}$, Tamas Varga ${ }^{1}$, Anil Krishna Battu ${ }^{1}$, Matthew A. Marcus ${ }^{2}$, Ondrej Krivanek $^{3}$, Tracy Lovejoy ${ }^{3}$ and Ravi Kukkadapu ${ }^{1}$

${ }^{1}$ Pacific Northwest National Laboratory, Richland, Washington, United States, ${ }^{2}$ Lawrence Berkeley National Laboratory, Berkeley, California, United States, ${ }^{3}$ Nion Co., Kirkland, Washington, United States

Although macro-scale in nature, most biogeochemical processes are driven by interactions at the microand nano-scale. Therefore, precise characterization of both organic and inorganic system components requires multi-scale, often multi-capability approaches. Here, we present the state-of-the-art characterization of organic matter-mineral associations by high-resolution correlated chemical imaging.

Microbially-produced extracellular polymeric substances (EPS), along with plant root exudates and their necromass are the primary source of organic matter $(\mathrm{OM})$ in soils. OM, composed mostly from biogenic components, can be either microbially transpired to $\mathrm{CO}_{2}$ and released back to the atmosphere, or via direct and indirect interaction with soil minerals can be tranformed to soil organic matter (SOM). Depending on its chemical makeup (aliphatic, aromatic, carboxylic, polysaccharide carbon compounds) and its localization within its immediate environment, it can be refractive (stabilized) or labile, with consequences to its resident time in soils (hours or days to years and centuries).

To improve our understanding of SOM biogeochemical interfaces that drive carbon stabilization in soils, we probed organo-mineral associations between SOM and minerals in a marginal soil from Prosser, WA using a suite of chemical imaging methods including STEM, EDS, scanning transmission X-ray microscopy with X-ray absorption near-edge structure (STXM/NEXAFS) and Energy electron loss spectroscopy (Aloof beam EELS) [1]. The over-reaching goal was to obtain spatially resolved imaging and spectral characterization of SOM-mineral interface at the micro- and nanoscale.

SOM is notoriously difficult to image and analyze by electron- and X-ray beam methods due to the sensitivity to the radiation damage inducing physical and chemical damage, such as material contraction during high-resolution analytical EM and soft X-ray imaging, and C-H compounds deposition during the STEM / EDS, as well as physical ablation by ionizing radiation. Cryo-microscopy conditions somewhat reduce radiation damage, but mitigating the effects of beam damage, while achieving artifact-free analytical results is one of the key objectives in the ongoing development of reliable, quantitative methods for chemical imaging.

For our experiment, the following workflow for imaging and analyses was established: First, a $120 \mathrm{kV}$ Tecnai Spirit BioTwin TEM (FEI) was used to identify regions of interest on a grid with microbial-mineral soil sample. Next, these exact locations were imaged and analyzed on a Nion aberration-corrected high energy resolution monochromated EELS-STEM (HERMES) at $60 \mathrm{keV}$ primary energy. Aloof EELS spectra were collected 30-50 nm away from the sample ROI's edge, with an energy resolution of $\sim 8 \mathrm{meV}$ at a beam current of $5 \mathrm{pA}$. Next, samples were transferred to ALS beamline 5.3.2.2 for STXM imaging and a coupled NEXAFS spectromicroscopy at the $\mathrm{C}, \mathrm{N}, \mathrm{O} \mathrm{K}$ edges and $\mathrm{Ca}$ and $\mathrm{Fe} \mathrm{L}$ edges on previously analyzed ROIs. Finally, the high- resolution STEM imaging was performed with a probe-corrected FEI Titan 80-300 operated at 300kV using HAADF detector. Selected area diffraction patterns of the ROI minerals were recorded with Gatan UltraScan1000 (2kx2k) CCD camera. Elemental mapping was performed with Oxford's $\mathrm{Si}(\mathrm{Li})$ detector and AZtec spectra analysis coupled to the STEM. 
Standards for each of the main organic carbon groups were also analyzed on each instrument: saccharides (trehalose), lipids (lauric acid), protein (BSA) and DNA, along with $\mathrm{CaCO}_{3}$ (calcite) mineral.

Correlated chemical imaging revealed distinct spatial and chemical correlation with $\mathrm{Ca}^{2+}$ mineral surfaces, substantiating the theory of 'Ca-OM bridges' crosslinking mechanism. The orchestrated workflow in this analytical sequence enabled obtaining analytical results with much lessened beam damage, completing the most structure-altering STEM/EDS as the final step. The resulting characterization of the Ca mineralSOM reactive surface composition in turn strengthened our understanding of processes at mineral-OM interfaces, relevant to the stability of SOM under environmental shifts. Ultimately, correlated chemical imaging can provide unprecedented insight into the interactions between SOM and minerals in a variety of environments.

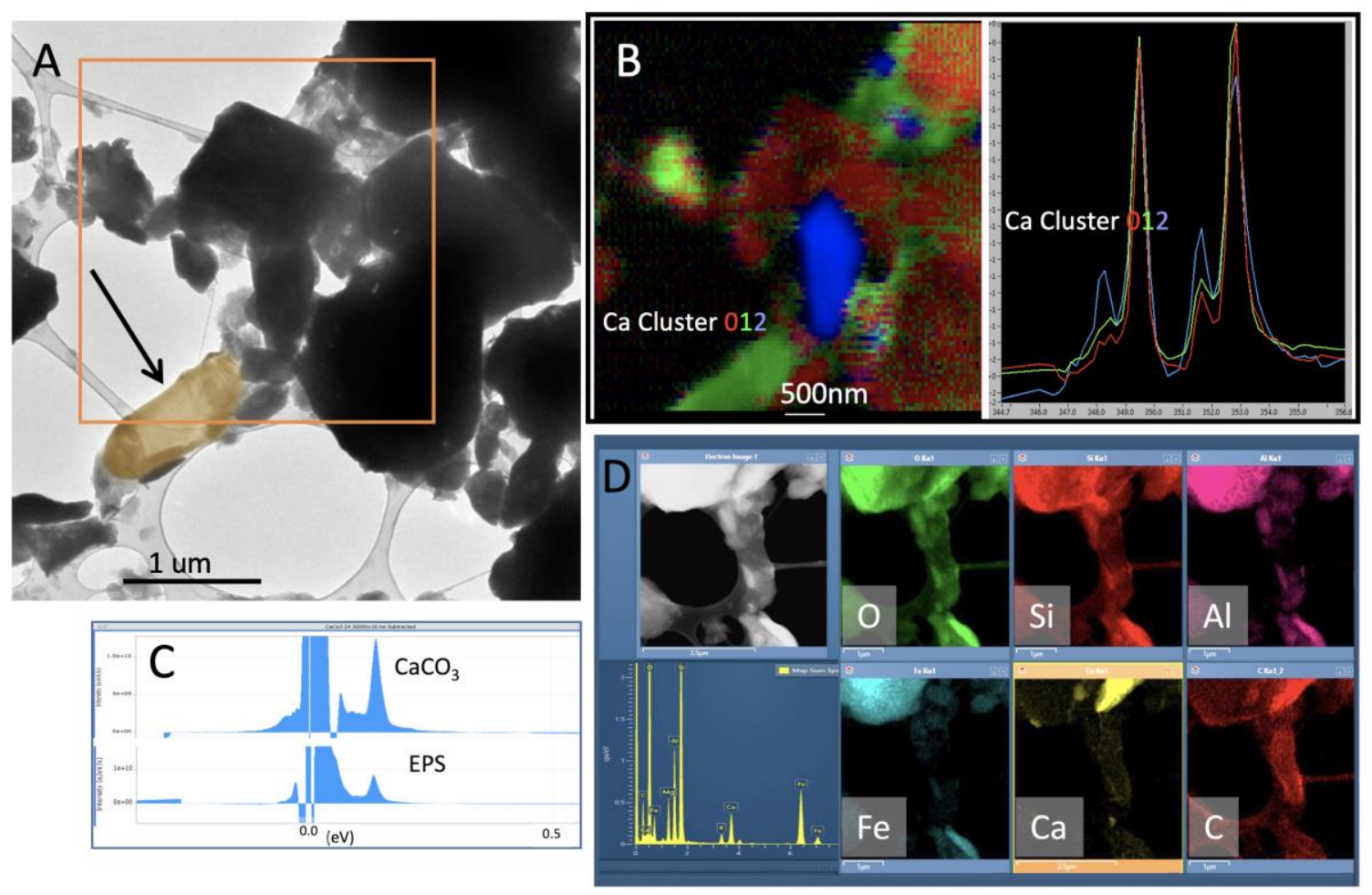

Figure 1. Correlated chemical imaging and spectroscopy. (A) TEM image of a typical soil microbe (colored) surrounded by EPS and minerals. (B) NEXAFS chemical map of area in inset (A). The organicrich areas correspond with spectra having low pre-peak intensities, suggesting disorder. Three species of Ca were localized correlating with C. (C) An example of EELS aloof data showing $\mathrm{C}$ signatures at the $<0.5 \mathrm{eV}$ range. (D) EDS map of the same area with visible $\mathrm{CaCO} 3$ and aluminosilicate minerals.

\section{References}

[1] Rez, P. et al. Damage-free vibrational spectroscopy of biological materials in the electron microscope. Nat. Commun. 7:10945 doi: 10.1038/ncomms 10945 (2016).

[2] This research was performed at the Environmental Molecular Sciences Laboratory (EMSL), a national scientific user facility sponsored by the Department of Energy's Office of Biological and Environmental Research, located at PNNL. STXM measurements were performed on beamline 5.3.2.2 at the Advanced Light Source, a DOE Office of Science User Facility under contract no. DE-AC02-05CH11231.

[3] The authors thank Dr. Janos Szanyi for obtaining FTIR spectra. 